\title{
A Method of Implementing Formalized Multidisciplinary Collaboration in Product Conceptual Design Process
}

\author{
YAN Xi-qiang ${ }^{\mathrm{a}, \mathrm{b}}$, LI Yan ${ }^{\mathrm{b},{ }^{*}}$, CHEN Jian ${ }^{\mathrm{c}}$, LI Wen-qiang ${ }^{\mathrm{b}}$, XIONG Yan ${ }^{\mathrm{b}}$ \\ ${ }^{a}$ China Aerodynamics Research and Development Center, Mianyang 621000,China \\ b School of Manufacturing Science \& Engineering, Sichuan University, Chengdu 610065,China \\ ${ }^{\mathrm{c}}$ Faculty of Engineering and the Environment, University of Southampton, Southampton SO17 1BJ, UK
}

\begin{abstract}
Modern product design can be considered as a process which involves intensive iteration, complex reasoning and mutual cooperation among the design groups in different disciplinary areas. In order to meet the requirements of modern products on multidisciplinary collaboration, the traditional method, where the product conceptual design is usually solved only within a single discipline, should be improved. Based on the classic FBS (Function-Behavior-Structure) model of product conceptual design, this paper studied the collaborative mechanism of functions, behaviors and structures, and proposed a new method/system of implementing formalized multidisciplinary collaboration in the product conceptual design process. In this method, the model/system of conceptual design process with multidisciplinary collaboration was established first, and three collaboration mechanisms were developed. After the coupling relationship among the design sub-states and the disciplinary classification characteristics of the design sub-states were expressed by the mathematical ways, the particle swarm algorithm was applied for the discipline planning, next the solving process for multidisciplinary collaboration were proposed. Finally, the conceptual design process of a stylus printer was used to illustrate the application of the proposed method.
\end{abstract}

Key words: multidisciplinary collaboration; discipline planning; product conceptual design; Function-Behavior-Structure

\section{Introduction}

There have been ever-increasing numbers of subsystems (disciplines) and components for modern products, such as hybrid vehicles, high-end printers, and medical equipment [1], and the design process of these products has become extremely complicated. Meanwhile, more and more new requirements and constraints, such as energy consumption and space restriction, have been put forward for the design of new products. Therefore, designers must consider the knowledge in various disciplines (e.g. kinematic, electrical, chemical, thermodynamic and electromagnetic) to satisfy these diverse requirements and constraints [2]. When the subsystems are integrated as a new product, however, the simple integration is not always an ideal solution and the desirable solution should consider the collaborative effect among the subsystems and realize the optimization in the whole product level [3-4].

The conceptual design of product originates from the user requirements which can be decomposed into functions one by one; next the individual function can be solved as corresponding behavior, and further solved as corresponding structure [5-6]. 
The behaviors and structures include not only geometrical information but also non-geometrical information, such as the relationship among the behaviors and among the structures [7]. From the perspective of system engineering, the product development process can be represented as a V-model [4, 8] (as shown in Figure1). In this model, conceptual design consists of the system-level definition of products, the hierarchical decomposition and definition of subsystems and components, and the integration of subsystems and components which are in line with the rules specified in different integration levels and verification phases. Therefore, there are mainly three tasks in the product concept design process:

- Identify the requirements and convert them into the system-level description about the designed products;

- Hierarchically decompose the system-level description into the subsystem description (component description) and realize the functions in individual subsystem as the corresponding behaviors and structures;

- Define the interface among the behaviors and among the structures from different subsystems, i.e. the collaborative relationship among subsystems.

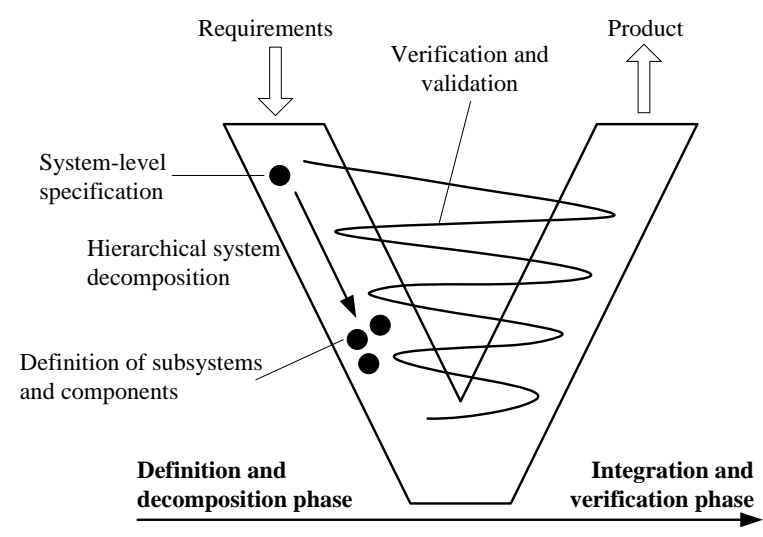

Figure1. V-model for product development process ${ }^{[4.8]}$

Traditionally, after the subsystems are obtained through the hierarchical decomposition, the solving of the corresponding behavior and structure is always carried out within individual subsystem and it is difficult to carry out the collaboration among different subsystems. As a result, the final integrated product will inevitably have redundant structures, and it is also impossible to design a product with overall optimization on the structure and performance. In order to obtain the optimal design scheme in the product conceptual design process, the collaborative relationship among different subsystems should be considered, and the collaboration among the functions, among the behaviors and among the structures should be established. In other words, the third task mentioned above is very necessary.

Up to now, much research has been contributed to the study of product conceptual design. Gero et al. [9-10] proposed a classic FBS (Function-Behavior-Structure) model and claimed that the process of conceptual design is a mapping process from functions to product structures. Based on the Gero's 
work, $\mathrm{Zu}$ et al. [11] developed an iterative principle based FBES (Function-Behavior-Mechanism-Structure) model and studied the relationship among the functions, behaviors, mechanisms and structures in the mechanical system. More recently, Christophe et al. [12] proposed a REBS (Requirement-FunctionBehavior-Structure) model and $\mathrm{Li}$ et al. [13] proposed a RPFSP (RequirementPrinciple-Function-Structure-Project) model. Feng et al. [14] put forward a model based on the cycle crossover solution of product functions, effects, working principles and structure projects and studied the mapping patterns and mapping relationship; Song el at.[15] established the logic connections among different types of basic mapping units and created a function solving framework by means of multilevel mixed mapping. Chen el at.[16,17] proposed a new model of conceptual design based on Scientific Ontology and intentionality theory. All of the studies mentioned above can support the product conceptual design process, but because the solving process is always limited to the knowledge in a single discipline, the multidisciplinary collaboration is restricted and it is difficult to obtain the best conceptual design scheme.

On the other hand, many researchers have actually investigated the significance of multidisciplinary collaboration during the product design process. Multidisciplinary Design Optimization (MDO) is an emerging methodology [18] and has been widely used in the design of complex products such as aircrafts [19], launch vehicles [20], satellites [21] and automobiles [22].Nevertheless, the MDO is currently mainly applied to the detailed design phase rather than the conceptual design phase. Because the principle scheme for the designed products has been determined in the conceptual stage, the designers cannot freely apply the multidisciplinary collaboration to obtain the best solution during the detailed design phase and the only thing that can be done is to optimize the shared variables among different disciplines in compromised ways. Therefore, the multidisciplinary collaboration in the detailed design phase can only obtain limited and partial optimization on the structure and performance of the designed products. The basic reason causing the aforementioned problem is that in the early design phase where the maximum design freedom is allowed, the designers always rely on their own limited knowledge and experience and it is unlikely for them to have an overall consideration on the collaborative effects among different disciplines.

Due to the high design freedom of the conceptual design phase, it is necessary to apply the MDO in the conceptual design phase rather than the detailed design phase to obtain the overall optimization on the structure and performance of the designed products. Meanwhile, because $75 \%$ of the final product cost is determined in the conceptual design phase [23], the implementation of multidisciplinary collaboration in the early phase can also save the market launching time and the cost of the products. This paper proposed a method to implement the MDO in the conceptual design phase. Based on the classic conceptual design model of functions, behaviors and structures, a method of implementing formalized multidisciplinary collaboration was developed for product conceptual design process. The multidisciplinary collaboration involves the coupling characteristics of different disciplines and the multidisciplinary 
collaborative relationship among the functions, behaviors and structures of the conceptual design. The proposed method consists of three steps. In the first step, based on the formalization representation of product conceptual design, the process with multidisciplinary collaboration is modeled; next, the particle swarm algorithm is used for the disciplinary planning; in the third stage, the solving mechanism is used to solve multidisciplinary collaboration and the finally obtain the best conceptual design scheme. It is worthy pointing out that in this paper, we were trying to formalize and model the multidisciplinary collaboration by mathematical forms or computer language forms, so the essential aim is that some programmable algorithms, artificial intelligent tools and digitalized knowledge repositories are able to use to assist the whole collaboration process in conceptual design stage, and finally we can realize the target of computer aided conceptual design in the future.

\section{Modeling the Conceptual Design Process with Multidisciplinary Collaboration 2.1 Formalization representation}

The process of product conceptual design starts from the user requirements, and can be considered as a conversion process from the function requirement to the structural scheme, and also with various design constraints [24]. The process can be represented as a quintuple form: $P=\{I, O, \Phi, \Gamma, \mathrm{B}\}$. As shown in Figure 2-a. I represents the input of a product design, such as design tasks or requirements; $O$ represents the output of the product design, i.e. the final conceptual design scheme. $\Phi$ represents the different design states and each design state set consists of design sub-states $\left(\Phi=\left\{\Phi_{1}, \Phi_{2}, \Phi_{3}, \cdots, \Phi_{n}\right\}\right) ; \quad \Gamma$ represents the transfer function mappings among the design states $\left(\Gamma=\left\{\Gamma_{1 \rightarrow 2}, \Gamma_{2 \rightarrow 3}, \Gamma_{1 \rightarrow 3}, \cdots, \Gamma_{i \rightarrow j}, \cdots\right\}, \quad(i \neq j, 1 \leq i \leq n, 1 \leq j \leq n)\right)$; $\mathrm{B}$ represents the feedback function with the repetition and iteration among the design states $\left(B=\left\{B_{2 \rightarrow 1}, B_{3 \rightarrow 2}, B_{3 \rightarrow 1}, \cdots, B_{j \rightarrow i}, \cdots\right\}, \quad(i \neq j, 1 \leq i \leq n, 1 \leq j \leq n)\right)$. As shown in Figure 2-a, the process of conceptual design can be also considered as a conversion process of the design states by both the transfer function $\Phi_{j}=\Gamma_{i \rightarrow j}\left(\Phi_{i}\right)$ and the repetition and iteration feedback function $\Phi_{i}=B_{j \rightarrow i}\left(\Phi_{j}\right)$ Therefore, the essential of the conceptual design process of products is the conversion process among design states.

The process of the product conceptual design with multidisciplinary collaboration is similar to the classic conceptual design process mentioned in the preceding section, and the critical difference is that the former process introduces multidisciplinary collaboration among design state subsets. In the traditional product conceptual design process, after the design state set is decomposed into design sub-states and the individual design sub-state is solved only in a single discipline, the overall state will be obtained by simply summing up of all subsets (if the solved 
subsets are denoted as $\Phi_{1}, \Phi_{2}, \ldots, \Phi \mathrm{n}$, so the overall state set is $\left.\Phi_{F}=\left\{\Phi_{1} \oplus \Phi_{2} \oplus \cdots \oplus \Phi_{n}\right\}\right)$. In contrast, in the process of the conceptual design with multidisciplinary collaboration, the overall state will be obtained with the consideration of the collaborative effects $\mathrm{C}$ among the sub-states set and it can be represented as $\Phi_{F}{ }^{\prime}=\left\{\Phi_{1} \otimes \Phi_{2} \otimes \cdots \otimes \Phi_{n}\right\}$, where $\Phi_{F}{ }^{\prime}=C\left(\Phi_{F}\right)$. Therefore, when the collaborative effect is taken into account, the formal representation of product conceptual design process can be change to a six-tuple form $P=\left\{I, O, \Phi^{\prime}, \Gamma, \mathrm{B}, C\right\}$ As

shown in Figure 2-b, $\Phi^{\prime}$ is the new design state which considers the collaborative effects among different disciplines.

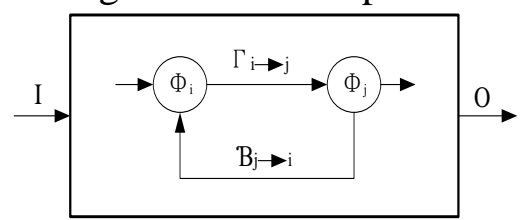

Figure2-a: Model of the traditional conceptual design

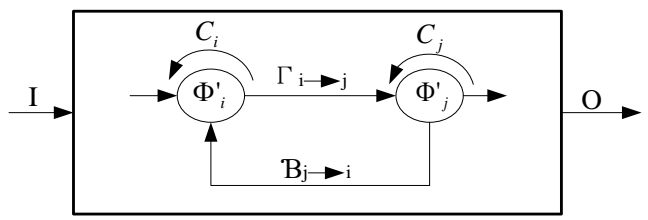

Figure2-b: Model of the conceptual design with multidisciplinary collaboration

Figure2. Formalization representation of the product conceptual design

\subsection{Collaboration process model}

As mentioned above, the essential of the product conceptual design process is the conversion process of the different design states, but the details of the conversion process are complicated and different conceptual design models have different design states and conversion strategies. In this paper, the product conceptual design process was modeled as three design states: functions, behaviors and structures. Function is the product attributes satisfying the users' requirements. Behavior is the physical principles and behavior approaches which realize the function. Structure is the description of the physical components or features of the design object. Based on the FBS mapping method by Umeda [25], the mapping mechanism of functions, behaviors and structures in the new developed model is established by using the mapping relations of the design feature sets from two adjacent layers. When the effects of multidisciplinary collaboration act on the mapping process, the designers will be guided to consider the collaborative effect among the sub-states of the functions, behaviors and structures.

Regarding the multidisciplinary collaboration in the function state, the relationship among different disciplines is built by means of the function flows, so as to realize the sharing and complementation among different disciplines. Taking the rotation motion in automobile as an example, it can fan the heat radiator of air condition system and warm the interior space of automobile, meanwhile under certain conditions (braking), it can generate electricity with the collaboration of other components. The multidisciplinary collaboration in the behavioral layer is realized through effects. For example, in the automobile braking system, the braking function 
is fulfilled by the behavior of braking, but the behavior of braking also influences the behavior of stability and safety of the automobile. Thus, it is necessary to consider the effect of braking behavior on the design of stability and safety. Because the design of the physical structure should meet the performance requirements of the products, in the structural layer the structural collaboration among different disciplines is realized by the couplings of parameters. For example, the design of automobile body involves various structural requirements, such as the structural strength, aerodynamics and human factors engineering (ergonomics), therefore the design scheme of the body should be the coupling results of these factors in different disciplines.

In summary, introducing the multidisciplinary collaboration into each design state of product conceptual design process is important to obtain the optimal and creative conceptual design scheme. The process of the product conceptual design with multidisciplinary collaboration is shown in Figure 3.

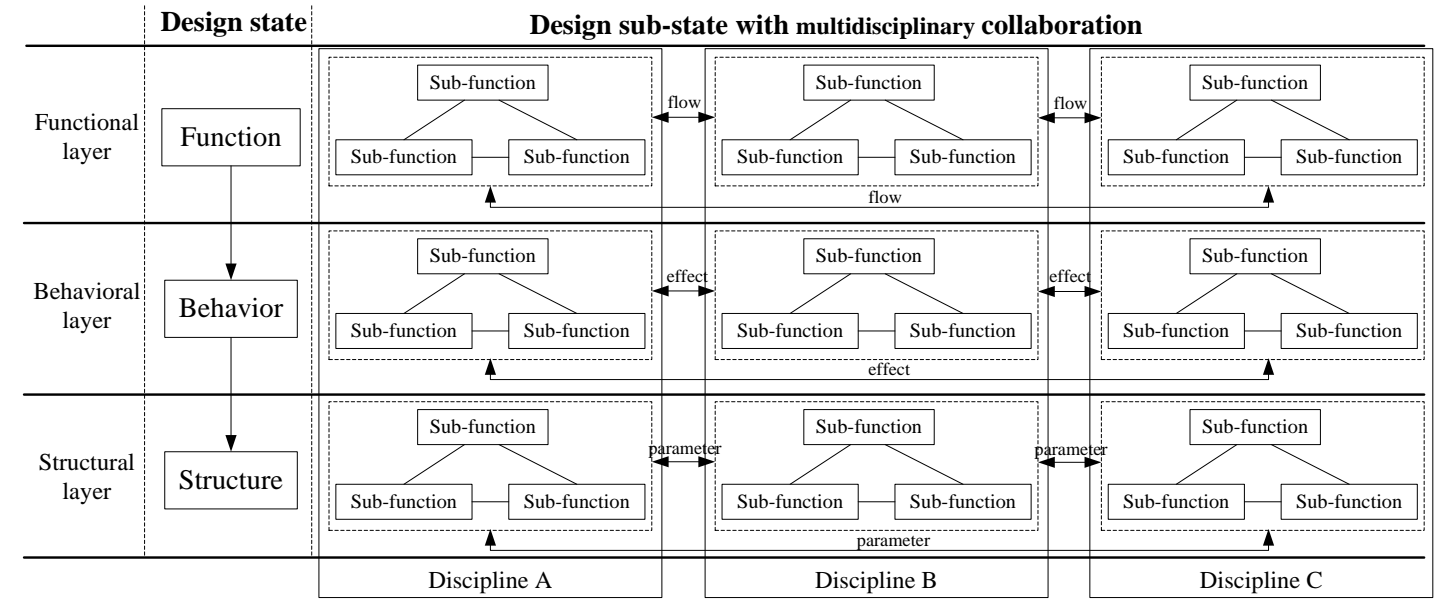

Figure3. Process of the product conceptual design with multidisciplinary collaboration

\subsection{Collaboration mechanisms}

As shown in the formalization representation, the collaboration effect is the critical factor for the conversion of design states and the output of the final overall state. Therefore the collaboration mechanisms among different disciplines in the conceptual design stage are very important. However, up to know, there is no any formalized collaboration mechanism to support the conceptual design process, but on the other hand, much research has been contributed to the collaboration mechanisms in the product detailed design process. These studies can be a foundation of developing new collaboration mechanisms for the conceptual design stage, so it is necessary to analyze these existing collaboration mechanisms. Ma [26] proposed that the product collaborative design mechanism can be fallen into three categories: interconnection, swap and interoperability. Chen et al. [27] proposed that the coupling factor of collaborative design could divide into three categories: the first one is only associated with the independent variable of the subsystem, the second one is only associated with other subsystems coupling variables, the third one is associated with both above. Tomiyama et al.[28] claimed that the complexity of 
multidisciplinary design comprises the complexity by design and the intrinsic complexity of multi-disciplinarity, and they also classified three types of relationships among theories[29]in regard to the complexity of multidisciplinary: two theories have relationships because of designation(instantiation) of entity, the two theories intrinsically share one common concept and two theories generate new concept. Based on the works in the above references, new formalized multidisciplinary collaboration mechanisms for the product conceptual design were proposed in this paper, including add-up collaboration, coupling collaboration and abrupt collaboration.

(1) Add-up collaboration is for the situation that a certain connection exists between two disciplines but no shared component (or parameter) between them. For this type of collaboration (as shown in Figure 4-a), two disciplines collaborate through their common linkages, without any new generation of component (or parameter). For example, the design of centrifugal compressors involves the relationship of fluid-thermal-solid, so the temperature parameter in the discipline of pneumatics needs to be transferred to the discipline of thermodynamics and the pressure and temperature parameters in the discipline of pneumatics will then influence the stress conditions in the static strength analysis.

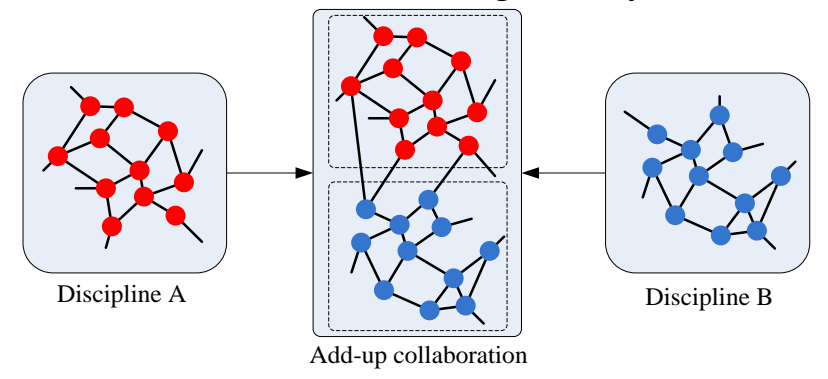

Figure 4-a. Scheme of add-up collaboration

(2) Coupling collaboration is for the situation that there are couplings and shared components (or parameters) between two disciplines (as shown in Figure 4-b). Two disciplines are closely connected through high degree of interaction. For example, when a conductor moves in the magnetic field and cuts the magnetic induction line, the force will be the parameter shared by the discipline of dynamics and the discipline of electromagnetics.

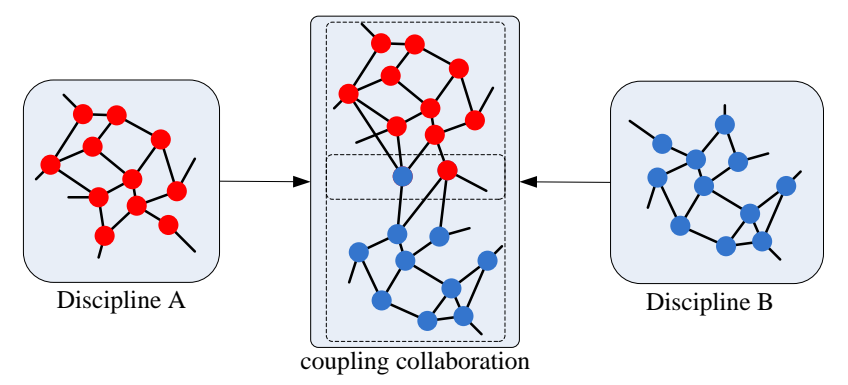

Figure 4-b. Scheme of coupling collaboration

(3) Abrupt collaboration is for the situation that there are strong couplings and shared components (or parameters) between two disciplines but the shared 
components (or parameters) are new generated components (or parameters) after couplings rather than those from direct couplings (as shown in Figure 4-c). For example, for the automobiles with manual transmission system, the gearbox provides various conversion ratios of speed and torque from the combustion engine to the differential through selecting different gear pairs, and the clutch is installed between the engine and the gearbox and manages the connection between them. The abrupt collaboration between the traditional clutch and gearbox enables the new design of automatic transmission system where a torque converter engages the transmission and a set of planetary gear provides a range of gear ratios.

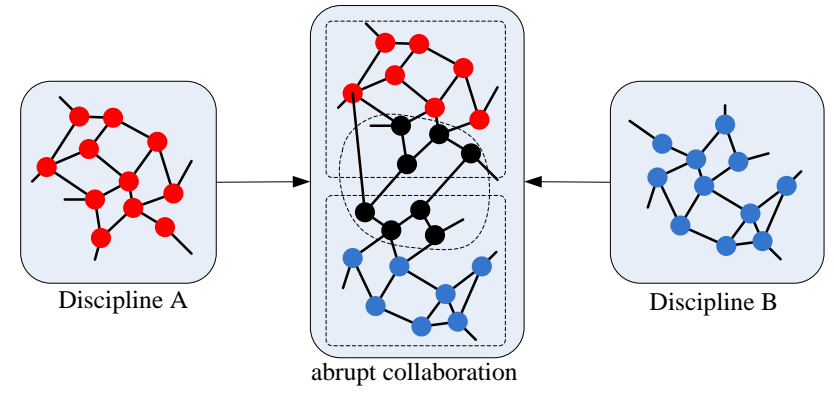

Figure 4-c. Scheme of abrupt collaboration

\section{Discipline Planning Method}

For the multidisciplinary collaboration in the product conceptual design process, the basic unit for the collaboration is discipline (subsystem).During the general product conceptual design process, the structure tree [30] is always used to represent the results from hierarchical decomposition. This up-to-down representation approach can directly show the hierarchy of design sub-states. However, in the conceptual design process of the multidisciplinary products, due to the complexity and coupling among different disciplines, the structure tree cannot efficiently represent the relationship of the importance and interdependence among the design sub-states. Therefore, after the user requirements are analyzed and converted into design states and each design state is further divided into design sub-states; meanwhile, it is necessary to carry out discipline planning on the design sub-states and classify the sub-states into different disciplines. Next, based on the discipline planning results, multidisciplinary collaboration is carried out on the design sub-states of the functions, behaviors and structures through the collaboration mechanisms proposed in the preceding section.

In the discipline planning, the degree of coupling among the design sub-states can be represented as a matrix $C$. The sub-states of the functions, behaviors and structures are semantic information rather than the mathematic information, so for the degree of coupling, the value definition of the elements in $C$ is based on the design experience of designers. If the coupling value of the sub-state $O_{i}$ to the sub-state $O_{j}$ is $c_{i j}$, and the coupling value of the sub-state $O_{j}$ to the sub-state $O_{i}$ is $C_{j i}$, most of the time there is a inequality for two values $c_{i j} \neq c_{j i}$ and this is because two sub-states have different dependencies for each other. If the total number of design sub-states is $q$, the full coupling matrix $\left[C_{q q}\right]$ can be written as: 


$$
C_{q q}=\left[\begin{array}{ccccc}
c_{11} & \cdots & c_{1 j} & \cdots & c_{1 q} \\
\cdots & & & & \cdots \\
c_{i 1} & & c_{i j} & & c_{i q} \\
\cdots & & & & \cdots \\
c_{q 1} & & c_{q j} & & c_{q q}
\end{array}\right]
$$

Meanwhile, the discipline planning also needs to classify the design sub-states into different disciplines. If the number of the design sub-states is $q$ and the number of the disciplines is $k$, the disciplinary classification matrix of the design sub-states about the disciplines can be written as a matrix $\left[X_{q k}\right]$ :

$$
X_{q k}=\left[\begin{array}{ccccc}
x_{11} & \cdots & x_{1 m} & \cdots & x_{1 k} \\
\cdots & & & & \cdots \\
x_{n 1} & & x_{n m} & & x_{n k} \\
\cdots & & & & \cdots \\
X_{q 1} & & x_{q m} & & x_{q k}
\end{array}\right],
$$

where, $x_{n m}=\left\{\begin{array}{l}1, \text { if the } n \text {th object belongs to discipline } m \\ 0, \text { if the } n \text {th object does not belong to discipline } m\end{array}\right.$

The target of the discipline planning is to calculate and obtain the optimal discipline classification matrix $\left[X_{q k}\right]$ ', which can satisfy the highest inner-discipline coupling and the lowest cross-discipline coupling. The constraint on solving the optimal discipline classification matrix is the constraint whether the disciplinary attributes are satisfied in the design process, or the principle that the designers judge whether the discipline classification on the sub-states is reasonable.

The inner-discipline coupling value indicates the coupling degree of the sub-states about a certain discipline. For discipline $l$, the inner-discipline coupling value $C_{l}$ can be calculated by:

$$
C_{l}=\left|C_{q q} X_{q l}\right|=\|\left[\begin{array}{ccccc}
c_{11} & \cdots & c_{1 j} & \cdots & c_{1 q} \\
\cdots & & & & \cdots \\
c_{i 1} & & c_{i j} & & c_{i q} \\
\cdots & & & & \cdots \\
c_{q 1} & & c_{q j} & & c_{q q}
\end{array}\right]\left[\begin{array}{l}
x_{1 l} \\
x_{n l} \\
x_{q l}
\end{array}\right]=\llbracket\left[\begin{array}{l}
\sum_{n=1}^{q} c_{1 n} x_{n l} \\
\sum_{n=1}^{q} c_{i n} x_{n l} \\
\sum_{n=1}^{q} c_{q n} X_{n l}
\end{array}\right]=\sum_{m=1}^{q} \sum_{n=1}^{q} c_{m n} x_{n l}
$$

If the total number of the disciplines is $\mathbf{k}$, the sum of the inner-discipline

coupling values can be calculated by: $f_{1}\left(x_{n p}, k\right)=\sum_{p=1}^{k} \sum_{m=1}^{q} \sum_{n=1}^{q} c_{m n} x_{n p}$

where, $\mathrm{p}=(1,2, \cdots, \mathrm{k})$.

The cross-discipline coupling value indicates the coupling degree of the sub-states about two different disciplines, and also indicates the feasibility of the 
collaboration between them. The coupling of disciplines is mainly caused by their shared sub-states. For discipline A and B, their shared sub-states can be expressed as a matrix $X_{q-A B}$, so the cross-discipline coupling values between disciplines A and B $\left(C_{A B}\right)$ can be written as:

$$
\begin{aligned}
& \left.C_{A B}=\left|C_{q q} X_{q-A B}\right|=\| \begin{array}{ccccc}
c_{11} & \cdots & c_{1 j} & \cdots & c_{1 q} \\
\cdots & & & & \cdots \\
c_{i 1} & & c_{i j} & & c_{i q} \\
\cdots & & & & \cdots \\
c_{q 1} & & c_{q j} & & c_{q q}
\end{array}\right]\left[\begin{array}{l}
X_{1 A} X_{1 B} \\
X_{n A} X_{n B} \\
X_{q A} X_{q B}
\end{array}\right]=\mid\left[\begin{array}{l}
\sum_{n=1}^{q} c_{1 n} X_{1 A} X_{1 B} \\
\sum_{n=1}^{q} c_{i n} X_{n A} X_{n B} \\
\sum_{n=1}^{q} c_{q n} X_{q A} X_{q B}
\end{array}\right] \\
& =\sum_{m=1}^{q} \sum_{n=1}^{q} c_{m n} X_{n A} X_{n B}
\end{aligned}
$$

If there are $k$ different disciplines, the number of the couplings among these disciplines is $N=C_{k}^{2}=\frac{k(k-1)}{2}$. So the sum of the cross-discipline coupling values of $k$ different disciplines can be calculated by

$$
f_{2}\left(x_{n a}, x_{n b}, k\right)=\sum_{a=1}^{\frac{k \bullet(k-1)}{2}} \frac{k \bullet(k-1)}{\sum_{b=1}^{2}} \sum_{m=1}^{q} \sum_{n=1}^{q} c_{m n} x_{n a} x_{n b}
$$

Therefore, the goal of discipline planning, in other words, is to achieve the maximum sum of the inner-discipline coupling values and the minimum sum of the cross-discipline coupling values. By combining Equation 1 and Equation 2, the mathematical expression for the planning goal can be written as:

$$
\begin{aligned}
& \text { Max } F\left\{f_{1}\left(x_{n p}, k\right),-f_{2}\left(x_{n a}, x_{n b}, k\right)\right\} \\
& \text { 其中 } f_{1}\left(x_{n p}, k\right)=\sum_{p=1}^{k} \sum_{m=1}^{q} \sum_{n=1}^{q} c_{m n} x_{n p} \\
& \qquad f_{2}\left(x_{n a}, x_{n b}, k\right)=\sum_{a=1}^{\frac{k \bullet(k-1)}{2}} \frac{k \bullet(k-1)}{\sum_{b=1}^{2}} \sum_{m=1}^{q} \sum_{n=1}^{q} c_{m n} x_{n a} x_{n b}
\end{aligned}
$$

In Equation 3, $q$ is the number of sub-states involved in the planning; $k$ is the total number of disciplines in the planning; $c_{m n}$ is the degree of coupling between the $m$ th sub-state and the $n$th sub-state; $x_{n p}$ is a disciplinary classification value of the $n$th sub-state to the discipline $p$. Because $x_{n p}$ is a discrete value from 0 to 1 , the mathematic optimization on the discipline classification matrix $\left[X_{q k}\right]$ can be considered as a typical 0-1 planning problem and the number of variables (elements) that need to be solved is uncertain. Based on the method proposed in reference [31], the $0-1$ planning problem can be equivalently converted into a mathematical problem 
with nonlinear complementary constraints. In this conversion, the Nonlinear Complementarity Problem (NCP) function can be used to transfer the $0-1$ variables into a corresponding non-smooth equation which can be smoothed by the Aggregate Function Method [32]. Consequently, the original optimization problem becomes a differentiable nonlinear planning problem which can be solved by the Particle Swarm Optimization algorithm(PSO) [33-34]. The flowchart of using PSO for the discipline planning is shown in Figure 5.

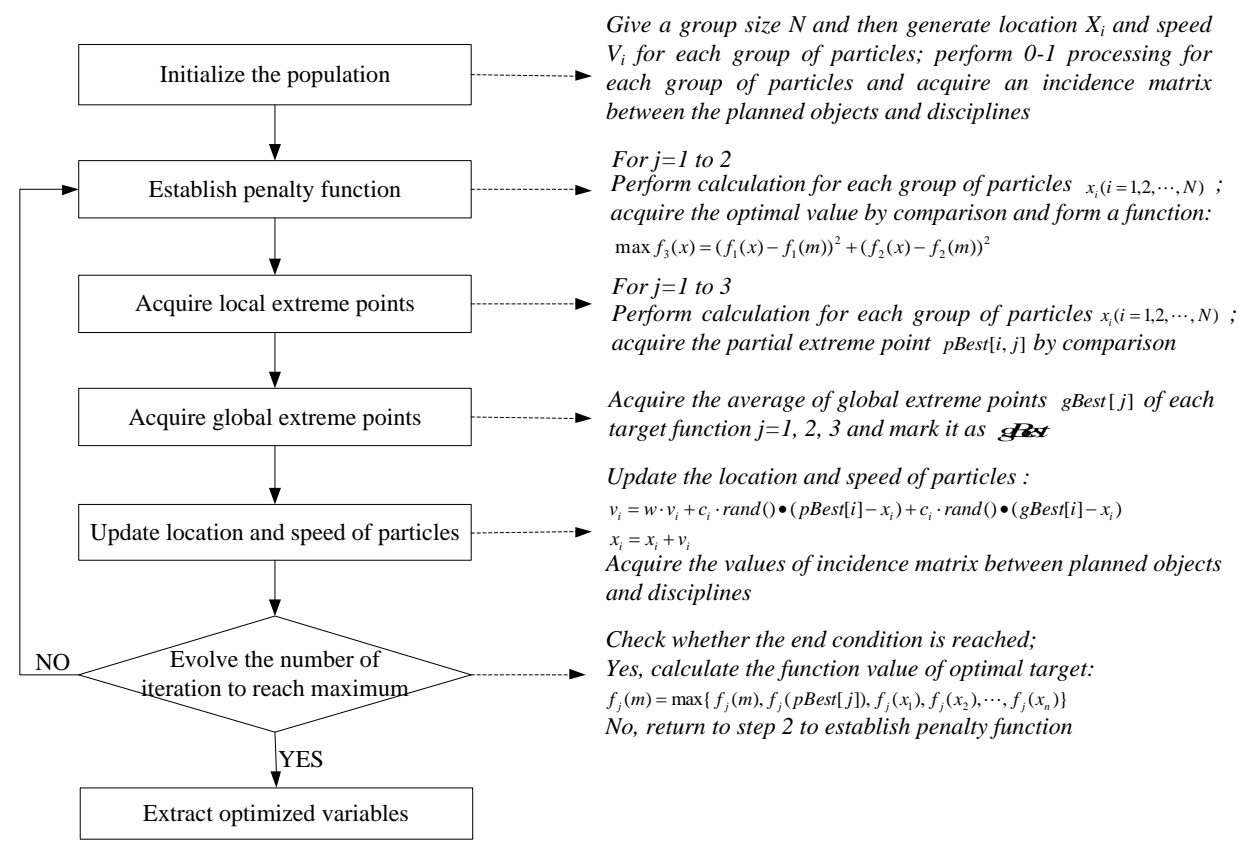

Figure 5. Discipline planning based on the PSO

\section{Solving on Multidisciplinary Collaboration}

The discipline planning is based on the FBS model shown in Figure 3, so the discipline planning for each state layer will be carried out step by step. Following discipline planning model, the design sub-states, which are the objects for the next step collaboration process, are categorized into different disciplines. Traditionally, even though the designers may build good discipline planning results, if there is no systematic collaboration model to support the next stage collaboration process, they are prone to carry out the collaboration based on their intuitive thinking (such as team brainstorming). Or some senior designers may resort their experience to solve collaboration, but this experience is always fragmentary. Most importantly, not all product conceptual designers have long-term accumulated design experience. Therefore, in this paper, we tried to formalize the collaboration process by using the three collaboration mechanisms proposed in Section 2.3, next some powerful knowledge systems are used to support the solving process of collaboration. It is worthy pointing out that our current effort is only trying to manually formalize the collaboration process (try to transform the inputs and outputs of the formalization into computer languages), but in the future the formalization can be carried out by an artificial intelligent system (such as fuzzy logic system). Finally, the formalized 
collaboration solving system can more efficiently and timely provide the best innovative scheme. The new developed multidisciplinary collaboration model is also based on the FBS model in Figure 3, so after the discipline planning in function layer, the function sub-states are imputed into the collaboration process and generate the next stage sub-states in behavior layer, then continue the discipline planning and collaboration in behavior layer and structure layer, finally output the conceptual design scheme. The process of the whole discipline planning and collaboration system is shown in Figure 6.

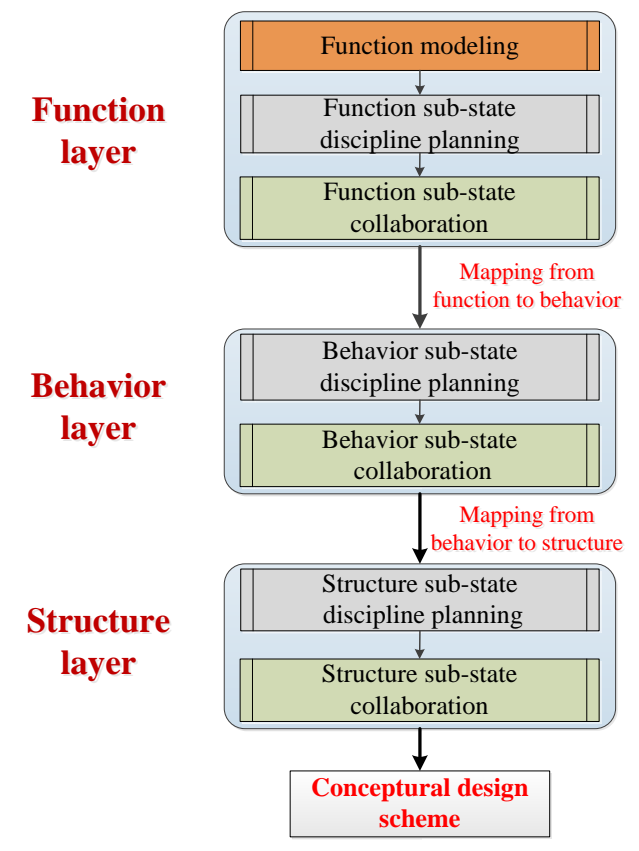

Figure 6. FBS model based discipline planning and collaboration system

After the discipline planning, the first question in the next stage is which design sub-states should be selected for the discipline collaboration? The answer is that the potential sub-states in one discipline should have strong correlation with the sub-states in other discipline. Similar to the coupling value in Section 3, we defined another coupling algorithm to evaluate the collaboration potentiality of each sub-state, but this coupling is based on the categorized disciplinary groups, so some coupling areas between/among different disciplines should be considered, such as

Fluid-structural interaction area between fluid mechanics discipline and solid mechanics discipline

Piezoelectric material area between mechanical discipline and electronic/electrical discipline

Quantum computing area between quantum physics discipline and computing discipline

It can be seen that this coupling areas normally are the new areas/sub-disciplines of collaboration. These coupling areas compose the top/abstract layer of the multidisciplinary knowledge repository, which will be discussed later on. Currently, 
the coupling evaluation needs much involvement of the designers' experience, but we are accumulating more and more discipline coupling areas into the repository/database (the designers can also input more effects into repository/database during their long term use of this model), so finally the system can become very powerful supporting tool for coupling value evaluation. Same as the definition in Section 3, if discipline A consists of $i$ number of design sub-state $\left\{a_{1}, a_{2}, \ldots, a_{i}\right\}$, and discipline $\mathrm{B}$ consists of $j$ number of design sub-state $\left\{b_{1}, b_{2}, \ldots, b_{j}\right\}$, the coupling value of each sub-state in the discipline $\mathrm{A}$ to the discipline $\mathrm{B}$ is given by

$$
C_{\mathrm{a}_{\mathrm{i}} \mathrm{B}}=\sum_{\mathrm{n}=1}^{\mathrm{j}} C_{\mathrm{a}_{\mathrm{i}} \mathrm{b}_{\mathrm{n}}}
$$

and the coupling value of each sub-state in the discipline B to the discipline $\mathrm{A}$ is given by

$$
C_{\mathrm{b}_{\mathrm{j}} \mathrm{A}}=\sum_{\mathrm{m}=1}^{\mathrm{i}} C_{\mathrm{a}_{\mathrm{m}} \mathrm{b}_{\mathrm{j}}}
$$

Based on the evaluated coupling values, the ranking for collaboration potentialities of the sub-states in discipline group A and B can be obtained; consequently we find the sub-states for the next stage collaboration.

\begin{tabular}{|c|c|c|c|}
\hline $\begin{array}{l}\text { Object } 1 \triangleleft \text { relation } \longrightarrow \text { Object } 2 \\
\text { Attribute } 1 \longleftrightarrow \text { Attribute } 2\end{array}$ & $\begin{array}{l}\text { Incidence } \\
\text { Relation }\end{array}$ & $\begin{array}{l}\text { Interactive } \\
\text { relation }\end{array}$ & $\begin{array}{l}\text { Independence } \\
\text { relation }\end{array}$ \\
\hline Same Attribute & add-up collaboration & coupling collaboration & abrupt collaboration \\
\hline Different Attribute & $\begin{array}{l}\text { add-up collaboration } \\
\text { abrupt collaboration }\end{array}$ & $\begin{array}{r}\text { coupling collaboration } \\
\text { abrupt collaboration }\end{array}$ & abrupt collaboration \\
\hline
\end{tabular}

Table 1. Collaboration mechanism selection

In fact, the collaboration solving process can be considered as, based on the attributes' comparison and the relations between/among the object states, using the collaboration methods (also including the knowledge repositories corresponding to each method) to find a new sub-state (with collaboration) to satisfy the design constrains. So we can standardize the collaboration by the set

Collaboration $=\{\langle$ state $>,<$ attribute $>,<$ relation $>,<$ constraint $\rangle,<$ method $>\}$ where state can be any sub-state in function, behavior and structure layer, Note that, in this set, the state has two form, one is input(can be two or more object sub-states) of the collaboration, and the other is the output next stage sub-states with collaboration. Attribute is the abstract characters of the object sub-states and currently we have defined different types of attributes for each design state layer, for instance 30 attributes (in the form of function basis [35]) for the function layer. Relation is the relationship between/among the object sub-states, and we classified all relations into three categories, incidence relation, interactive relation and independence relation. Constraint includes all items that final output sub-state has to satisfy with, mainly including some common categories, such as cost, performance, reliability and so on, and we also tried to use computer readable languages (in the form of the input of 
fuzzy logic algorithm) to represent these constrains, such as low, medium, high, slow, fast etc.., Method is actually the three mechanisms proposed in Section 2.3. So based on the outputs of discipline planning, the system selects the potential sub-states as the objects of collocation (as mentioned above), and the conceptual designers input some information (or select from provided categories/parameters) about the attributes, and relations, then the system selected the suggested collaboration mechanism (as shown in Table 1). There may be more than one collaboration results (candidates sub-states in next layer or design scheme in final step) from each collaboration, so the designers has to use the constrains to find the best one. So it can guarantee that the final design scheme always satisfy the product design constrains.

In order to support the solving of these collaboration mechanisms, four kinds of knowledge repositories are provided. The inputs of searching include the involved disciplines, sub-states' attributes, and some keywords about the design target (such as printer, wind tunnel, electric vehicle etc.). The knowledge repositories are presented in many kinds of forms, but all of them include the abstract design sub-states information (by keywords or short phases) which was extracted during the repositories' establishment stage. So the knowledge searching can efficiently find the relevant knowledge by advanced search algorithms. If the designers click this information, more details can be found, such as text description, figure, animation etc. After the relevant knowledge (abstract information) is found from the knowledge repositories, all the abstract information items of found knowledge are considered as the nodes of Figure 4, then the system analyzes theses nodes (based on the attributes and relations of the nodes), chooses some nodes, and uses the selected mechanisms to generate the next stage sub-states (or final scheme) with disciplinary collaboration. Currently the designers need to involve the collaboration process of the found knowledge by the guide of the selected collaboration mechanism, but our ultimate target is, in the future, to intelligently carry out this collaboration process based on the abstract information, such as using the automatic semantic processing technology [36, 37].

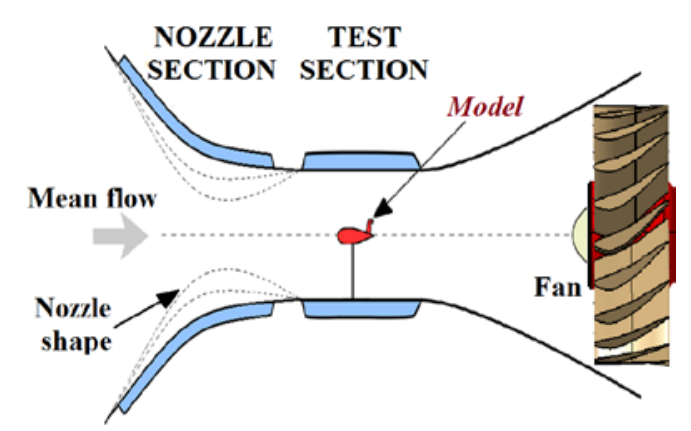

Figure 7. Wind tunnel configuration and nozzle shape

We introduce the conceptual design (in function stage) of a wind tunnel's nozzle as an example to illustrate the collaboration mechanism selection and collaboration solving process. The main design requirement for the wind tunnel nozzle is to generate a mean air flow at a required axial speed (Mach number). As shown in Figure 7, according to the well-known continuity and Bernoulli equations, when the 
nozzle shape changes, the cross sectional area changes, the air flow speed subsequently increases or decreases. In order to satisfy the requirement of different flow speed in different experiments, a group of functions can be defined, following the discipline planning and based on the calculated coupling values by Eqs.(4\&5), we selected two functions to do collaboration, "nozzle shape adjusting" (different adjusting levels to generate different flow speeds, only based on the classic fluid mechanics equations) and "nozzle wall fixing" (the wall should be firm and rigid, with minimal structural vibration). The function sub-state "nozzle shape adjusting" belongs to aerodynamic discipline, and the function sub-state "nozzle wall fixing" belongs to structural discipline. The flow chart of whole collaboration process is shown in Figure 8. The attributes of sub-states "nozzle shape adjusting" and "nozzle wall fixing” are "change state" and "fasten object” respectively. There is an incidence relation between two sub-states. Based on the Table 1, we obtained the selection results of collaboration mechanism: add-up and abrupt. Next, the system inputted “aerodynamic, structural, change state, fasten object, nozzle" into the knowledge repositories for searching and found out some abstract design information.

1. cutting the wall into sections

2. adjustable conical nozzle with an opening and a cylindrical tube

3. imparting motion to the aerodynamic body by an interaction between aerodynamic surface and fluid flow

4. adjusting the flexible walls of a wind tunnel

5. detachable air flow concentrating nozzle from housing

As mentioned above, clicking these information items, more details can be found, such as item 1 from the "cut" rule of TRIZ invention principle and item 2 from patent US3949321. Afterward the selected collaboration processed the found information to generate three candidate sub-states for the next stage (behavior stage) with collaboration. Finally, the constrains (low cost, long term use, easy to install and replace, light weight, up to 1.5 Mach high flow speed) were inputted into system and the designer decided if the new sub-state has satisfied these constrains. 


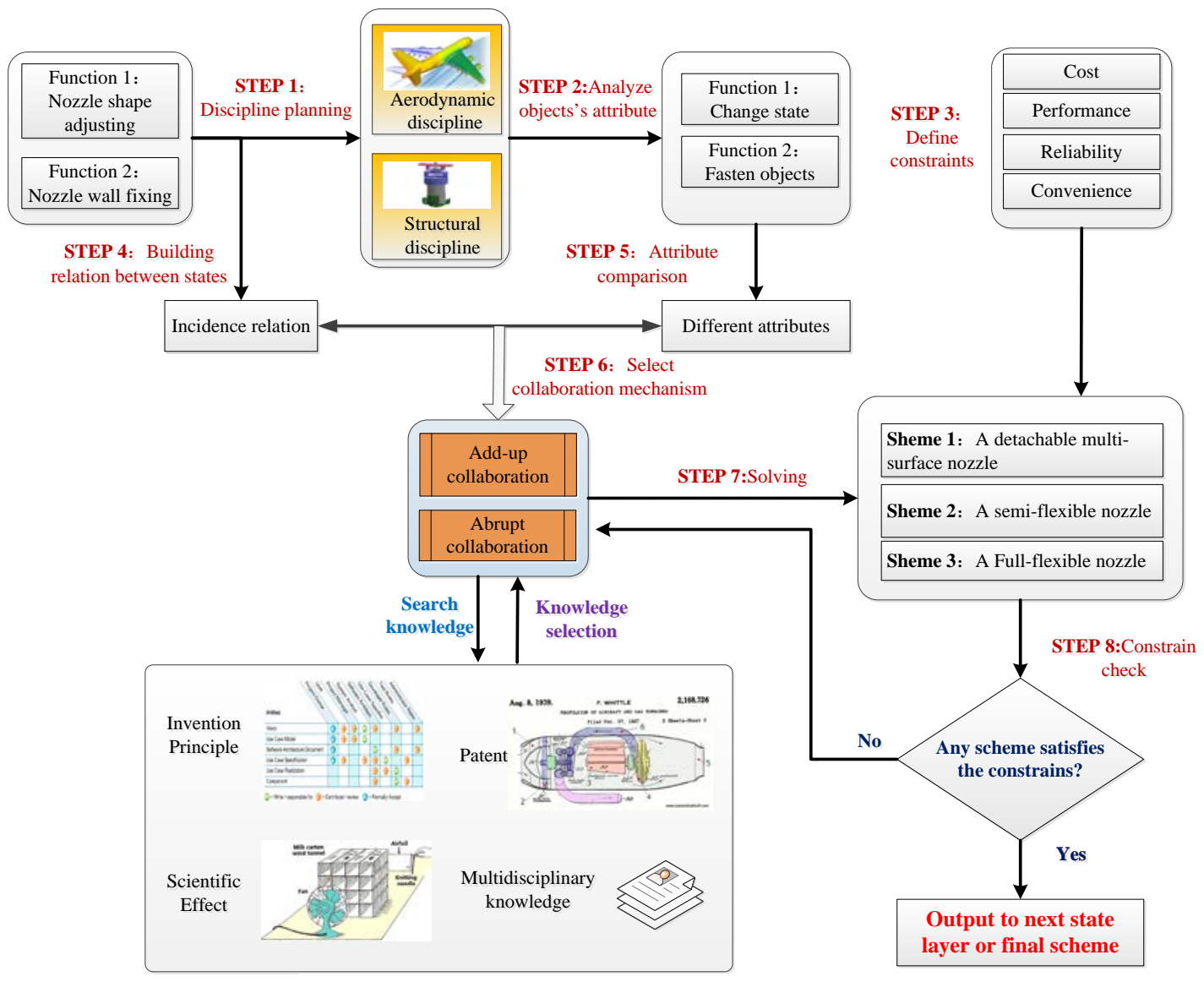

Figure 8. Flowchart of the function collaboration on nozzle design

Based on the solving process described above, it can be found that the knowledge repository is a critical element in the process. The Product Design Group in Sichuan University is trying to build a comprehensive knowledge repository and knowledge searching program. So in this paper, we will only briefly introduce these knowledge repositories below and more details on our accomplishments in this topic can be found in Refs.[38-40]. Because there are different types of knowledge, the knowledge repository can be divided into: invention principle knowledge repository, patent knowledge repository, scientific effect knowledge repository and multidisciplinary knowledge repository. The knowledge in the invention principle knowledge repository explains the abstract TRIZ invention principle (Theory of Inventive Problem Solving proposed by G. S. Altshuler[41] ) by means of real application cases. The knowledge in patent knowledge repository contains a wide variety of patents which have been collected and processed. Scientific effect knowledge repository includes the knowledge of a specific scientific phenomenon which existed indifferent areas, and this knowledge is described by the means of text, images, video and animation. The multidisciplinary knowledge repository contains more specific knowledge in multiple areas, such as mechanical, electrical, control, etc. As mentioned above, the multidisciplinary knowledge repository include two layers, the first layer consists of the abstract coupling areas and the second layer consists of more specific information, including 8 parts: conceptual description, working principles, collaboration attribute, 
advantages, application examples, technological areas, reference information and related knowledge.

After carrying out the collaboration in three design state layers step by step, the final outputs from the collaboration in the structure layer should be still abstract. In order to transform these abstract outputs into the specific structural design, the designers need involve more in this final scheme generation stage. During this process, all knowledge items which have been found in the previous collaboration steps can be easily accessed and read by the designers. The various representations (text, images, video and animation) of the knowledge can assist the designers in conceiving new product design scheme. Meanwhile the designers can also use their experience to adjust the final scheme and obtain the optimal results.

\section{Case Study}

Based on the new product conceptual design method proposed above, the conceptual design process of a certain type of stylus printer is introduced here to illustrate the implementation of the method. Following the disciplinary analysis on the systematic configuration, the printer can be divided into four different subsystems: mechanic subsystem, electric subsystem, control subsystem and thermodynamics subsystem.

(1) Function modeling and planning. The functions of the stylus printer were first modeled by the Function Analysis Methods proposed by Pahl and Beitz [42]. Next the coupling values of each pair of functions were evaluated, but the coupling relationship between the functions (also same for the coupling relationship between the behaviors and between structures) could not be obtained quantitatively, so the coupling values were subjectively evaluated by a group of designers. In order to avoid the evaluation distortion from the subjectivity of the designers, the evaluated coupling values from different designers were averaged as the final coupling value. Afterwards, the full coupling matrix $\left[C_{q q}\right]$ was determined. In the coupling matrix $\left[C_{q q}\right.$ ], the value of each element indicates the degree of coupling ( 9 for strong coupling, 7 for ordinary coupling, 5 for weak coupling degree and 1 for very weak coupling). The full coupling matrix of the functions of the stylus printer is shown in Table 2 (design inputs). According to the coupling characteristics among these functions, 21 functions were classified into 4 disciplines (subsystems), i.e. mechanics, electrics, control and thermodynamics, and consequently the disciplinary classification matrix $\left[X_{q k}\right]$ of these functions about 4 disciplines were formed. The disciplinary classification matrix is shown in Table 3 (design inputs). After the coupling matrix and disciplinary classification matrix were decided, based on the discipline planning method, the problem was mathematically modeled and the particle swarm algorithm was used to perform the discipline planning on the functions. As mentioned above, the result of discipline planning is the optimal discipline classification matrix $[X q k]$ ', therefore the optimal classifications of the 21 functions about the 4 disciplines were finally obtained and shown in Table 4 (the planning results are inputs of the next stage disciplinary collaboration).The detail of the calculation process can be found in our former publication[43]. 
Table 2: Coupling matrix of the functions of the stylus printer

\begin{tabular}{|c|c|c|c|c|c|c|c|c|c|c|c|c|c|c|c|c|c|c|c|c|c|c|}
\hline \multicolumn{2}{|c|}{ Function coupling } & 1 & 2 & 3 & 4 & 5 & 6 & 7 & 8 & 9 & 10 & 11 & 12 & 13 & 14 & 15 & 16 & 17 & 18 & 19 & 20 & 21 \\
\hline 1 & Power start & & 9 & 9 & 9 & 9 & 1 & 1 & 7 & 7 & 7 & 7 & 1 & 1 & 1 & 7 & 1 & 9 & 9 & 9 & 9 & 9 \\
\hline 2 & Power input & 1 & & 9 & 9 & 9 & 1 & 1 & 7 & 7 & 7 & 7 & 1 & 1 & 1 & 7 & 1 & 9 & 9 & 9 & 9 & 9 \\
\hline 3 & Power adjustment & 1 & 1 & & 7 & 7 & 1 & 1 & 1 & 1 & 1 & 1 & 1 & 1 & 1 & 7 & 1 & 1 & 1 & 1 & 1 & 1 \\
\hline 4 & Torque conversion & 1 & 1 & 1 & & 7 & 1 & 1 & 1 & 7 & 7 & 7 & 1 & 1 & 1 & 7 & 1 & 1 & 1 & 1 & 1 & 1 \\
\hline 5 & Motion transfer & 1 & 1 & 1 & 1 & & 1 & 1 & 7 & 7 & 7 & 1 & 1 & 1 & 1 & 7 & 1 & 1 & 1 & 1 & 1 & 1 \\
\hline 6 & Speed switch & 1 & 1 & 1 & 1 & 1 & & 7 & 7 & 5 & 5 & 5 & 5 & 5 & 5 & 5 & 5 & 1 & 1 & 1 & 1 & 1 \\
\hline 7 & Paper placement & 1 & 1 & 1 & 1 & 1 & 1 & & 7 & 5 & 5 & 5 & 5 & 5 & 5 & 5 & 5 & 1 & 1 & 1 & 1 & 1 \\
\hline 8 & Paper transporting & 1 & 1 & 1 & 1 & 1 & 1 & 1 & & 5 & 5 & 5 & 5 & 5 & 5 & 5 & 5 & 1 & 1 & 1 & 1 & 1 \\
\hline 9 & Paper cutting & 1 & 1 & 1 & 1 & 1 & 1 & 1 & 1 & & 5 & 9 & 9 & 1 & 1 & 5 & 5 & 1 & 1 & 1 & 1 & 1 \\
\hline 10 & Paper positioning & 1 & 1 & 1 & 1 & 1 & 1 & 1 & 1 & 5 & & 9 & 9 & 1 & 1 & 5 & 5 & 1 & 1 & 1 & 1 & 1 \\
\hline 11 & Vertical movement & 1 & 1 & 1 & 1 & 1 & 1 & 1 & 1 & 1 & 1 & & 9 & 1 & 1 & 5 & 5 & 1 & 1 & 1 & 1 & 1 \\
\hline 12 & Horizontal movement & 1 & 1 & 1 & 1 & 1 & 1 & 1 & 1 & 1 & 1 & 1 & & 9 & 9 & 5 & 5 & 1 & 1 & 1 & 1 & 1 \\
\hline 13 & Stylus-tip striking & 1 & 1 & 1 & 1 & 1 & 1 & 1 & 1 & 1 & 1 & 1 & 1 & & 5 & 5 & 5 & 1 & 1 & 1 & 1 & 1 \\
\hline 14 & Stylus-tip positioning & 1 & 1 & 1 & 1 & 1 & 1 & 1 & 1 & 1 & 1 & 1 & 1 & 1 & & 5 & 5 & 1 & 1 & 1 & 1 & 1 \\
\hline 15 & Printing and coloring & 1 & 1 & 1 & 1 & 1 & 1 & 1 & 1 & 1 & 1 & 1 & 1 & 1 & 1 & & 5 & 1 & 1 & 1 & 1 & 1 \\
\hline 16 & Drying medium & 1 & 1 & 1 & 1 & 1 & 1 & 1 & 1 & 1 & 1 & 1 & 1 & 1 & 1 & 1 & & 1 & 1 & 1 & 1 & 1 \\
\hline 17 & Impurity cleaning & 1 & 1 & 1 & 1 & 1 & 1 & 1 & 1 & 1 & 1 & 1 & 1 & 1 & 1 & 1 & 1 & & 1 & 1 & 1 & 1 \\
\hline 18 & Motion control & 1 & 1 & 1 & 1 & 9 & 9 & 1 & 1 & 1 & 9 & 9 & 9 & 9 & 9 & 1 & 1 & 1 & & 1 & 1 & 1 \\
\hline 19 & Signal control & 9 & 9 & 9 & 9 & 9 & 1 & 1 & 1 & 1 & 1 & 1 & 1 & 1 & 1 & 1 & 1 & 1 & 1 & & 1 & 1 \\
\hline 20 & Temperature control & 1 & 1 & 1 & 1 & 1 & 1 & 1 & 1 & 1 & 1 & 1 & 1 & 1 & 1 & 9 & 9 & 1 & 1 & 1 & & 1 \\
\hline 21 & Position control & 1 & 1 & 1 & 1 & 1 & 1 & 9 & 9 & 9 & 9 & 9 & 9 & 1 & 9 & 1 & 1 & 1 & 1 & 1 & 1 & \\
\hline
\end{tabular}

Table 3: Disciplinary classification matrix of the functions of the stylus printera

\begin{tabular}{|c|c|c|c|c|c|c|c|c|c|c|c|c|c|c|c|c|c|c|c|c|c|c|}
\hline \multicolumn{2}{|c|}{$\begin{array}{l}\text { Functions } \\
\text { Disciplines }\end{array}$} & 1 & 2 & 3 & 4 & 5 & 6 & 7 & 8 & 9 & 10 & 11 & 12 & 13 & 14 & 15 & 16 & 17 & 18 & 19 & 20 & 21 \\
\hline $\mathrm{I}$ & Mechanical subsystem & 0 & 0 & 0 & 1 & 1 & 1 & 1 & 1 & 1 & 1 & 1 & 1 & 0 & 1 & 1 & 1 & 1 & 0 & 0 & 0 & 0 \\
\hline II & Electrical subsystem & 1 & 1 & 1 & 1 & 1 & 0 & 0 & 0 & 0 & 0 & 0 & 0 & 0 & 0 & 0 & 0 & 0 & 0 & 0 & 0 & 0 \\
\hline III & Control subsystem & 0 & 0 & 0 & 0 & 0 & 0 & 0 & 0 & 0 & 0 & 0 & 0 & 0 & 0 & 0 & 0 & 0 & 1 & 1 & 1 & 1 \\
\hline IV & Thermodynamic subsystem & 0 & 0 & 0 & 0 & 0 & 0 & 0 & 0 & 0 & 0 & 0 & 0 & 0 & 0 & 1 & 1 & 0 & 0 & 0 & 0 & 0 \\
\hline
\end{tabular}

Table 4: Results of discipline planning on the functions of the stylus printer

\begin{tabular}{|c|l|l|}
\hline S/N & Discipline name & Functions included in the discipline \\
\hline I & Mechanical subsystem & $\begin{array}{l}\text { paper placement, paper transporting, paper cutting, paper positioning, vertical movement, } \\
\text { horizontal movement, stylus-tip striking, stylus-tip positioning, printing and coloring }\end{array}$ \\
\hline II & Electrical subsystem & power start, power input, power adjustment, torque conversion, motion transfer, speed switch \\
\hline III & Control subsystem & motion control, signal control, temperature control, position control \\
\hline IV & Thermodynamic subsystem & printing and coloring, drying medium \\
\hline
\end{tabular}

(2) Multidisciplinary collaboration and mapping of functions. Based on the 
results of the discipline planning on the functions and the selection results of potential sub-states and collaboration mechanism, the add-up collaboration, coupling collaboration and abrupt collaboration were applied to optimize different function sub-states of printer. The add-up collaboration between the "power input" of the electrical subsystem and the "medium drying" of the thermodynamic subsystem enables the use of electric power to dry the medium. The coupling collaboration was carried out between the "torque conversion" of the mechanical subsystem and the "conveying of medium" of the electrical subsystem and consequently the "transfer of substance or energy" became the shared function of both disciplines. Another coupling collaboration was carried out between the "stylustip positioning" of the mechanical system and the "position control" of the control system through the common parameter "position". The abrupt collaboration was carried out between the "vertical movement and horizontal movement" of the mechanical subsystem and the "motion control" of the control subsystem through the shared parameter "speed". According to the FBS model, the functions with multidisciplinary collaboration need to be mapped to the behaviors, and it is a process to acquire the physical principle and behavior serving as the basis of function realization. Meanwhile, the influence of the existing behaviors on new behaviors also needs to be considered in the mapping process. The mapping of the functions to the behaviors of the stylus printer is shown in Figure. 9.

\begin{tabular}{|c|c|c|c|}
\hline Functions & \multicolumn{2}{|c|}{ Function collaboration } & $\longrightarrow$ behaviors \\
\hline 1.Power start & & & 1. Circuit closing \\
\hline 2.Power input & & & 2.Transmission of electric field \\
\hline 3.Power adjustment & & & 3.Electromagnetic phase shift \\
\hline 4.Torque conversion & (1) & Transfer force & 4.Energy conservation \\
\hline 5.Motion transfer & & & 5.Squeezing/friction \\
\hline 6.Speed switch & & & 6.Drive ratio change \\
\hline 7.Paper placement & & & 7.Spatial position constraint \\
\hline 8.Paper transporting & & $\begin{array}{c}\text { Energy } \\
\text { conversion }\end{array}$ & 8.Friction force \\
\hline 9.Paper cutting & & (electric- & 9.Pressure \\
\hline 10.Paper positioning & & thermal) & 10.Refection of light \\
\hline 111.Vertical movement & & & 11.Drive force \\
\hline 12.Horizontal movement & & & 12.Vibration \\
\hline 13.Stylus-tip striking & & Cnod & 13.Constraint on position \\
\hline 14.Stylus-tip positioning & & ореел & 14.Absorption effect \\
\hline 15.Printing and coloring & & & 15.Heating/ absorption effect of light \\
\hline 16.Drying medium & & & 16.Substance separation \\
\hline 17.Impurity cleaning & & & 17.Speed sensing \\
\hline 18.Motion control & $\oplus$ & Location & 18.Conductivity/photoelectric valve \\
\hline 19.Signal control & & & 19.Thermosensitive substance \\
\hline 20.Temperature control & & & 20.Position measuring \\
\hline 21.Position control & & & \\
\hline
\end{tabular}

Figure 9. Mapping relationship between the functions and behaviors of the stylus printer 
(3) Multidisciplinary planning, collaboration and mapping of behaviors. Similar to the discipline planning on the functions, it is necessary to first consider the coupling effects among the obtained behaviors and establish the disciplinary classification matrix of the behaviors. Next, the particle swarm algorithm was used to perform discipline planning on the behaviors. The results of the discipline planning on the behaviors of the stylus printer are shown in Table 5. Following the discipline planning, and based on the collaboration mechanism selection method in Section 4, different collaboration mechanisms were applied to the multidisciplinary collaborations of the selected behavior sub-states in different disciplines. The abrupt collaboration between the "vibration" of the mechanical subsystem and the "electromagnetic phase shift" of the electrical subsystem created new "electromagnetic vibration". The add-up collaboration between the "transmission of electric field" of the electrical subsystem and the "drive force" of the mechanical subsystem enables the force in electric field function as the external force of the mechanical subsystem. Again, according to the FBS model, after the multidisciplinary collaboration, the behaviors need to be mapped to the structures so as to obtain the physical objects or the mechanisms of product design. Likewise, it is also necessary to consider the influence of the existing structures on the new structures during the mapping process. The results of the mappings from the behaviors to the structures of the stylus printer are shown in Figure 10.

Table 5: Outcome of the discipline planning on the behaviors of the stylus printer

\begin{tabular}{|l|l|l|}
\hline S/N & Discipline name & Behaviors included in the discipline \\
\hline I & Mechanical subsystem & $\begin{array}{l}\text { energy conservation, squeezing/friction, drive ratio change, spatial position } \\
\text { constraint, friction force, pressure, drive force, vibration, substance } \\
\text { separation, position measuring }\end{array}$ \\
\hline II & Electrical subsystem & circuit closing, transmission of electric field, electromagnetic phase shift \\
\hline & Control system & absorption effect, thermo-sensitive substance, conductivity \\
\hline IV & Thermodynamic system & refection of light \\
\hline
\end{tabular}




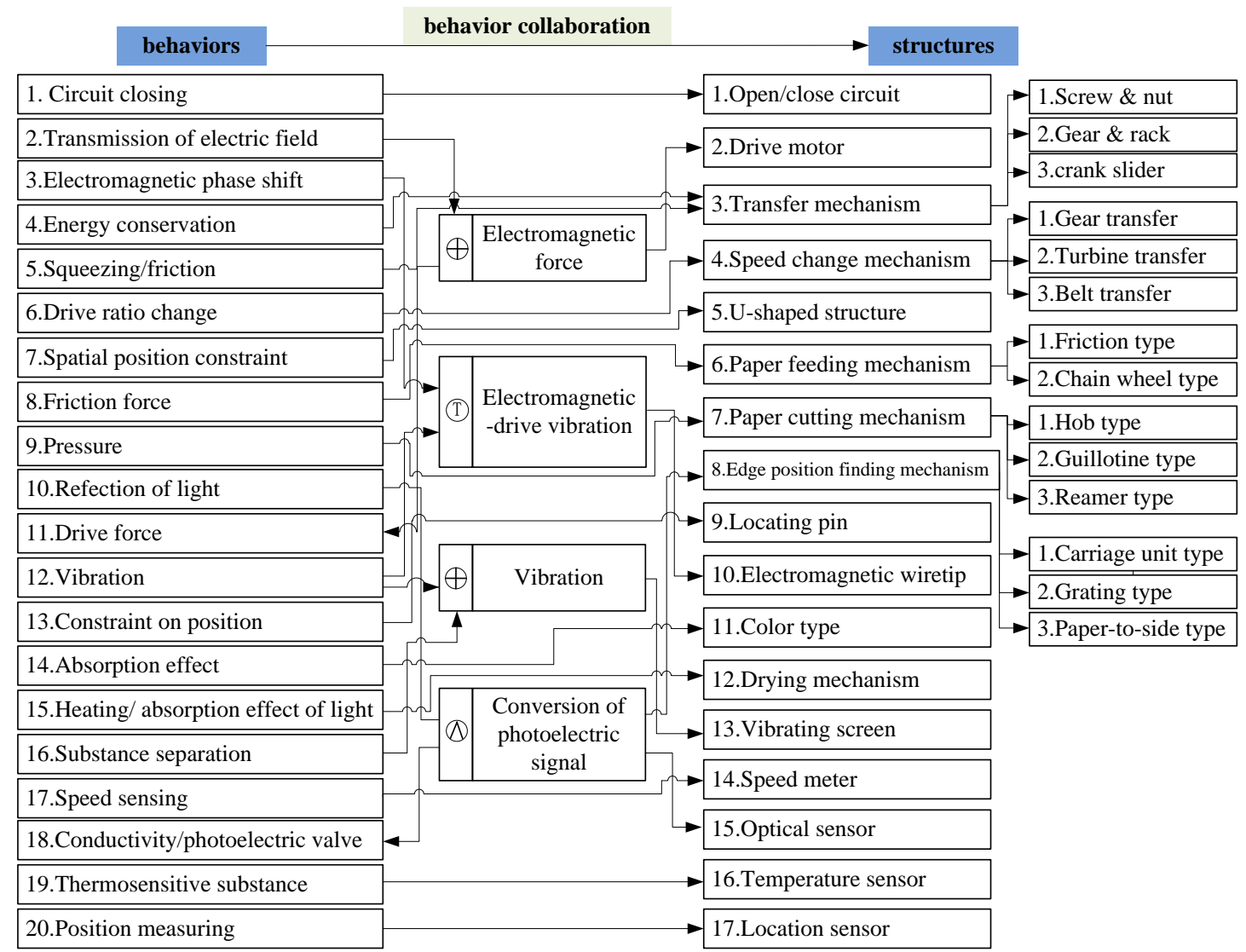

Figure 10. Mapping from the behaviors to the structure of the stylus printer

(4) Multidisciplinary planning and collaboration of structure. Similar to planning and collaboration of the functions and behaviors, it is necessary to consider the coupling of the structures of the stylus printer and establish the coupling matrix of the structures and the disciplinary classification matrix of the structures. The optimal disciplinary classification of the structures about the four different disciplines is shown in Table 6. Next, based on the collaboration mechanism selection method in Section 4, different collaboration mechanisms were applied to the multidisciplinary collaboration of the selected structure sub-states. The add-up collaboration between the "speed change mechanism" of the mechanical subsystem and the "speed meter" of the control subsystem transmits the speed of the "speed change mechanism" into the "speed meter". The add-up collaboration between the "paper feeding mechanism" of the mechanical subsystem and the "position sensor" of the control subsystem transmits the position information of the "paper feeding mechanism" into the "position sensor".

Table 6: Outcome of discipline planning for structures of stylus printer

\begin{tabular}{|c|c|c|}
\hline $\mathbf{S} / \mathbf{N}$ & Discipline name & Structures included in the discipline \\
\hline I & Mechanic system & $\begin{array}{l}\text { transfer mechanism, speed change mechanism, U-shaped structure, } \\
\text { paper advance mechanism, paper cutting mechanism, edge-finding } \\
\text { mechanism, locating pin, color tape, vibrating screen }\end{array}$ \\
\hline
\end{tabular}




\begin{tabular}{|l|l|l|}
\hline II & Electric system & open/close circuit, drive motor, electromagnetic print stylus \\
\hline III & Control system & speed meter, optical sensor, position sensor \\
\hline IV & Thermodynamics system & drying mechanism, temperature sensor \\
\hline
\end{tabular}

(5) Output of the conceptual design scheme of the stylus printer. As mentioned in Section 4, it can be found that the final outputs from the collaboration in the step (4) are still abstract. Therefore, more "human-force" has to be involved into the process of transforming these abstract outputs into the specific structural design. By checking and reviewing the knowledge item involved in the above collaboration process, finally we can figure out the final conceptual design of the stylus printer. As shown in Figure 11-2, compared with the design scheme without the considerations of multidisciplinary collaboration (as shown in Figure 11-1), the main changes can be found from the following three aspects: (1) An electromagnetic print head replaces the old mechanical print head and this change simplifies the structure and improves the efficiency and reliability of the printer; (2) The vibration generated by the pin striking on the ribbon is used as the power source to clear the impurities on the surfaces of the papers after printing, so using existing functions of the system improves the energy efficiency of the printer; (3) By the reasonable design on the spatial layout, the heat which is generated during the printing process and wasted in the old printer is used to dry the printing papers, consequently the old drying mechanism is removed in the new design.

It is worth pointing out that based on the case study of stylus printer, the application of proposed method and its implementation process has been illustrated and in overall the final results met the expedited goals of multi-disciplinary collaboration in the conceptual design phase, but due to some limitations of the knowledge repository, the support of knowledge repository was not good. The limitations of knowledge repository include the quantity limitation, quality limitation, searching method and so on. We have constantly made an effort to develop the knowledge models and the knowledge repository since 2008. So far, there are 5850 knowledge items in our knowledge repository, including 1450 invention principles, 2250 scientific effects and 2150 multidisciplinary knowledge items. But as shown in the case study, the knowledge repository still needs a lot of works in the future. We are continuously working on the building of knowledge repository no and the number of knowledge item is constantly increasing as well. Definitely the knowledge repository will become a powerful tool to support the conceptual design method proposed in this paper. 


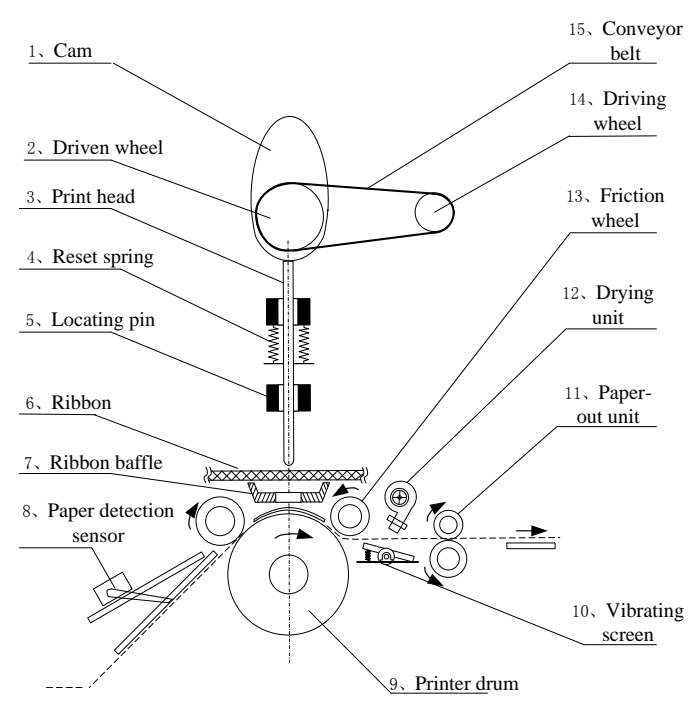

Figure 11-1. Old design scheme of the stylus printer

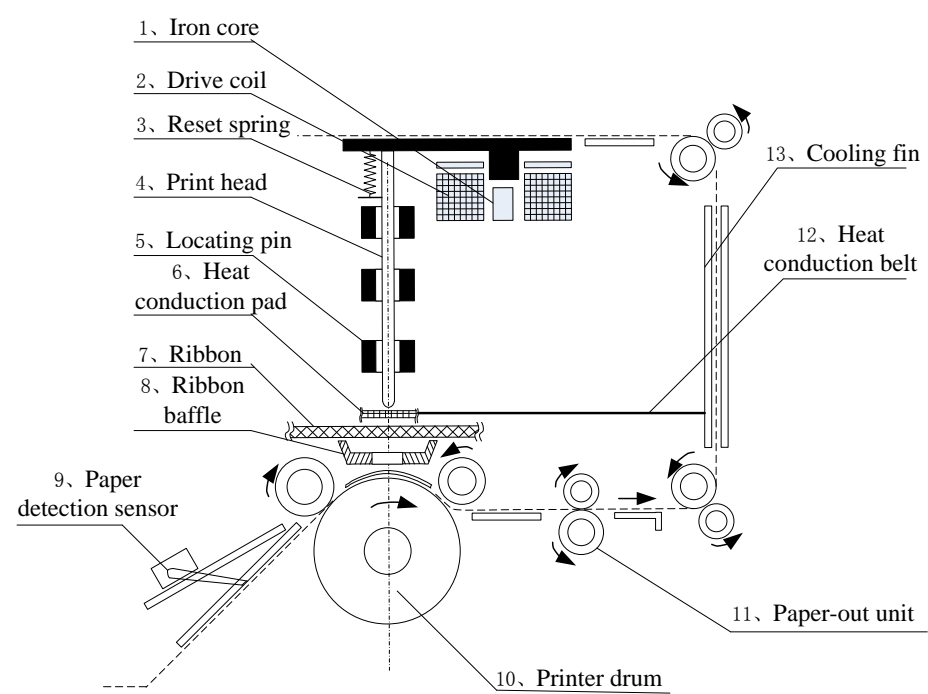

Figure 11-2. New conceptual design of the stylus printer with multidisciplinary collaboration

\section{Conclusion}

Because most part of the product cost is determined in the product conceptual design stage, it is more important to consider the multidisciplinary collaboration in the product conceptual design stage rather than in the product detailed design stage. Based on the classic FBS conceptual design model, this paper proposed a method which systematically integrated the multidisciplinary collaboration in the product conceptual design and can assist the designers to obtain the optimal and creative design schemes. The model of conceptual design with multidisciplinary collaboration was studied and three types of collaboration mechanism were defined as well. In order to carry out the multidisciplinary collaboration, the discipline planning on the design sub-states is necessary. In the discipline planning, based on the coupling matrix and 
discipline classification matrix of the design sub-state, the optimal discipline classification matrix can be calculated by the particle swarm algorithm. Next, multidisciplinary collaboration can be solved by the solving mechanism proposed in this paper and the conceptual design scheme with multidisciplinary collaboration can be obtained. Finally, the application of the proposed method was illustrated by the conceptual design process of a stylus printer and the innovation of the method has been demonstrated.

\section{Acknowledgements}

This work has been supported by the National Natural Science Foundation (Grant No. 51435011) and Innovation Method Fund of China (Grant No. 2013IM030500). The authors would like to thank the anonymous reviewers for their helpful and constructive comments that greatly contributed to improving the final version of the paper.

\section{References}

[1]. Goel AK, Rugaber S, Vattam S. Structure, behavior, and function of complex systems: the structure, behavior, and function modeling language. Artificial Intelligence for Engineering Design, Analysis and Manufacturing. 2009,23(1):23-35

[2]. CHEN Liang, JIN Guodong. Product modeling for multidisciplinary collaborative design. International Journal of Advanced Manufacturing Technology.2006.30(7):589-600

[3]. Tomiyama T, D Amelio V, Urbanic J, et al. Complexity of multidisciplinary design. CIRP Annals-Manufacturing Technology. 2007,56(1):185-188

[4]. Hitoshi Komoto,Tetsuo Tomiyama. A framework for computer-aided conceptual design and its application to system architecting of mechatronics products. Computer-Aided design. 2012,44(10):931-946

[5]. Chmarra MK, Cabrera AAA, van Beek T, et al. Revisiting the divide and conquer strategy to deal with complexity in product design. In: Proceedings, IEEE/ASME international conference on mechatronic and embedded systems and applications. 2008,393-398

[6]. Yehuda E. Enhancing multi-disciplinary collaboration through semantically rich representation. Automation in Construction. 2001,10:741-755

[7]. Pahl G, Beitz W. Engineering design: a systematic approach. New York: Springer Verlag. 1988

[8]. Forsberg K, Mooz H. The relationship of systems engineering to the project cycle. Engineering Management Journal. 1992,4(3):36-43

[9]. Gero J S. Design prototypes: a knowledge representation schema for design.AI Magazine. 1990,11(4):26-36

[10].Gero J S, Kannengiesser U. The situated function-behavior-structure framework. Design Studies. 2004,25(4):373-391

[11].ZU Y, XIAO R-bin, LIU Yong. New iteration-based conceptual design model of complex mechanical products. Chinese Journal of Mechanical Engineering. 2006,42(12): 197-205(in Chinese)

[12].F. Christophe, A. Bernarda, É. Coatanéa. RFBS: A model for knowledge representation of 
conceptual design. CIRP Annals-Manufacturing Technology. 2010,59 (1): 155-158

[13].LI Wen-qiang, LI Yan, WANG Jie, et al. The process model to aid innovation of products conceptual design. Expert Systems with Applications. 2010,37 (5): 3574-3587

[14].FENG Pei-en, ZHANG Shuai, PAN Shuang-xia. Cyclic solving process and realization for conceptual design of complex function product. Chinese Journal of Mechanical Engineering, 2005,41(3): 135-141

[15].SONG Hui-jun, LIN Zhi-hang. Hierarchical function solving framework with hybrid mappings in the conceptual design of mechanical products. Chinese Journal of Mechanical Engineering, 2003,39(5): 82-87

[16].CHEN Yong, ZHANG Zhi-nan, XIE You-bai et al. A new model of conceptual design based on Scientific Ontology and intentionality theory. Design Studies, 2015,37:12-36

[17]. CHEN Yong, LIU Ze-lin, XIE You-bai et al. A knowledge-based framework for creative conceptual design of multi-disciplinary systems. Computer-Aided Design, 2012,44, 146-153

[18]. Sobieski J.S, Haftka R.T. Multidisciplinary aerospace design optimization: Survey of Recent Developments. Structure and Multidisciplinary Optimization. 1997,14(1):1-23

[19].D.P. Raymer. Enhancing aircraft conceptual design using multidisciplinary optimization. PhD thesis, Royal Institute of Technology,2002

[20].J.Roshanian, Z. Keshavarz. Effect of variable selection on multidisciplinary design optimization: A flight vehicle example. Chinese Journal of Aeronautics. 2007,20(1):86-96

[21].M. Ebrahimi, M. Farmani, J. Roshanian. Multidisciplinary design of a small satellite launch vehicle using particle swarm optimization. Structural and Multidisciplinary Optimization. 2011,44 (6):773-784

[22].J.E. Park, F. Luis, A. Suleman. Multidisciplinary design optimization of an automotive magnetorheological brake design. Computers and Structures . 2008,86(3-5): 207-216

[23].Lotter B. Manufacturing Assembly Handbook, Butterworths, Bostom,1986

[24].LI Wen-qiang. The researches on the key techniques and methods for the creative Processes of product conceptual design. $P h D$ thesis, Sichuan University. 2009,24-25

[25].Umeda.Y, Masaki.I, Tomiyama.T, et al.Supporting conceptual design based on the function-behavior-state modeler. Artificial Intelligence for Engineering, Design, Analysis and Manufacturing . 1996,10(4):275-288

[26].MA Jun.Research and realization of key technologies in product collaborative design. $P h D$ thesis, Xi'an University of Technology. 2005, 10-11

[27].CHEN Bohong, XIAO Renbin, LIU Jihong. Research of the coupled factors in MDO for complex products.Chinese Journal of Mechanical Engineering. 2001, 37(1):19-23

[28].Tomiyama T.Knowledge Structure and Complexity of Multi-Disciplinary Design, Proc. 16th Int CIRP Design Seminar, 2006, 310-316.

[29]. Tomiyama, T. Knowledge Deployment: How to Use Design Knowledge, Human Behavior in Design-Individuals, Teams, Tools, Springer, Berlin, 2003, 261-271.

[30].CHEN Z. Similarity measurement based on decomposition of complex object. Computer Engineering and Applications. 2008, (34): 149-151+162

[31].LI Xin-si, TAN Tao. Continuous approach to discrete structural optimization. Chinese Journal of Applied Mechanics. 2006,24(3): 26-31

[32].Li Xing-si.An Entropy-based Aggregate Method for Minimax Optimization. Engineering Optimization. 1992, 18: 277-285. 
[33].Eberhart R, Kennedy J. A new optimizer using particles swarm theory. Proc. of the 6 th International Symposium on Micro Machine and Human Science, Nagoya, Japan. 2001, 39 43.

[34].Eberhart R, Shi Y. Comparing inertia weights and constriction factors in particle swarm optimization. Proc. of the Congress on Evolutionary Computation. 2000, 84 - 88.

[35]. Stone B. R. and Wood L. K. Development of a Functional Basis for Design. Journal of Mechanical Design, 2000, 144(4):359-370

[36]. Sowa F. J. Principles of Semantic Networks: Explorations in the Representation of Knowledge.Frame Problem in Artificial Intelligence, 1991,95-107

[37].González G. R. A Semantic Web approach to Digital Rights Management, Ph.D. Thesis, Universitat Pompeu Fabra, Spain, 2005

[38].FAN Fan, LI Yan, LI Wenqiang. Design knowledge organization and application strategy research based on FBS. Journal of Machine Design. 2012, 29 (10) :9-15

[39].LI Zipeng, LI Yan, XIE Aitong. EW knowledge model design and application based on knowledge engineering. Chinese Journal of Engineering Design. 2014, 32(4): 52-58

[40].TU Jianwei, LI Yan, LI Wenqiang. Knowledge retrieval model and implementation for product innovative design. Computer Integrated Manufacturing system. 2013, 19 (2): 300-308

[41].G.S.Altshuller. And suddenly the inventor appeared. Technical Innovation Center, INC, Worcester,1996

[42].Pahl G, Beitz W. Engineering Design-—A Systematic approach (2nd ed). London; Springer-Verlag,2000

[43].YAN Xi-qiang, LI Yan, LI Wenqiang. A planning method of decoupling based on coupling strength and FDSM of MDO. Computer Integrated Manufacturing system. 2013, 19(7):1447-1455 